\title{
L'intervention archéologique de 2004 dans les galeries nord et est du cloître de l'abbaye de Caunes-Minervois
} Arnaud Gaillard

\begin{abstract}
The existing cloister of the Caunes-Minervois abbey is a very sober work realised by the monks of the Congregation of St Maur at the end of the XVIIth century. The special nature of the site lies in the presence of remains of the medieval cloister, indicated by a paved stratum under the level of the Maurist cloister (-2,40 m approximately). Prior to alteration works, the excavations carried out in 20004 aimed at studying the remains of the medieval cloister. This operation brought to light a large part of the medieval north gallery and a consequent portion of the east one. Together with previous soundings, these include almost every part of the medieval cloister in the area of the existing cloister. However, certain questions still remain unanswered. We are unable to locate the medieval south gallery. At the very most, we can propose to place it under a modern béal crossing the convent according to a globally east-west axis. The discovery of a Romanesque semicircular arch in the alignment of the south arm of the transept seems to us a decisive element for the understanding of the building. This access associated with a fist threshold situated under the caladé level of circulation seems to indicate that an older (Romanesque) pavement may have been in use before the construction of the herringbone pavement. As a consequence, the age of the partly destroyed medieval small stone wall observed in the north gallery would be proved. As regards the relative chronology of the site, the evidences brought to light allow us to advance that the herringbone pavement (calade) belongs to the Gothic phase of reconstruction of the church nave.
\end{abstract}

\section{Citer ce document / Cite this document :}

Gaillard Arnaud. L'intervention archéologique de 2004 dans les galeries nord et est du cloître de l'abbaye de CaunesMinervois. In: Archéologie du Midi médiéval. Supplément n6, 2010. L’abbaye et le village de Caunes-Minervois (Aude). Archéologie et Histoire. pp. 57-67;

doi : 10.3406/amime.2010.1956

http://www.persee.fr/doc/amime_1278-3358_2010_sup_6_1_1956

Document généré le 14/03/2016 


\title{
L'intervention archéologique de 2004 dans les galeries nord et est du cloître de l'abbaye de Caunes-Minervois
}

\author{
Arnaud GAILLARD*
}

Le cloître actuel de l'abbaye de Caunes-Minervois est un ouvrage très sobre réalisé par les moines de la congrégation de Saint-Maur à la fin du XVII ${ }^{\mathrm{e}}$ siècle. La particularité du site réside dans la présence de vestiges du cloître médiéval, matérialisé par un sol caladé sous le niveau du cloître mauriste actuel (-2,40 m environ).

En préalable à des travaux d'aménagements, l'opération de fouille menée en 2004 avait pour objectif l'étude des vestiges du cloître médiéval. Cette opération a permis de mettre au jour une grande partie de la galerie nord médiévale et une portion conséquente de la galerie est. Ces sondages, associés aux précédents, comprennent la quasi-totalité des parties du cloître médiéval incluses dans l'aire du cloître actuel. Pour autant, certaines questions restent toujours sans réponses. Ainsi, nous ne sommes pas en mesure de localiser la galerie sud médiévale ; tout au plus, nous pouvons envisager de la situer sous un béal moderne traversant le cloître selon un axe globalement est-ouest. La découverte d'un arc en plein cintre roman dans l'alignement du bras de transept sud nous paraît être un élément déterminant pour la compréhension de l'édifice. Cet accès associé à un premier seuil situé sous le niveau de circulation caladé semble indiquer qu'un sol plus ancien (roman) ait pu fonctionner avant la mise en place du sol en épis. Par conséquent, l'ancienneté du mur bahut médiéval en partie épierré, observé dans la galerie nord, serait avérée. En ce qui concerne la chronologie relative du site, les indices mis au jour nous permettent d'avancer que le sol en épis (calade) appartient à la phase gothique de reconstruction de la nef de l'église.

Mots-clefs : Moyen Âge, Epoque moderne, Caunes-Minervois, Abbaye bénédictine, Cloître, Sol en épis.

\section{INTRODUCTION}

Comme l'a souligné notre collègue Olivier Ginouvez (Ginouvez et alii 2010), l'enquête archéologique consacrée à l'histoire de l'abbaye de Caunes-Minervois est intimement liée aux travaux de réhabilitation du monument. En effet, dans le droit-fil des actions menées par la commune depuis une quinzaine d'années, un important programme de restauration a été engagé en 2004. L'idée principale de ce projet de mise en valeur mené par Monsieur Régis Martin (ACMH) repose sur la relative superposition de deux cloîtres (Ginouvez 2000), permettant de créer un couloir en sous-œuvre de la partie nord-est du cloître actuel et offrant ainsi des possibilités de circulation propices à tous les scénarios de visite de l'abbaye.

Il était donc envisagé de créer "une galerie sous la galerie" dans la partie où les vestiges sont attestés avec une bonne continuité de présentation, c'est-à-dire depuis l'angle nord-ouest du cloître actuel, toute la galerie nord, et une portion de la galerie est en retour, environ jusqu'à l'escalier monumental (fig. 1). Une intervention archéologique s'avérait nécessaire au vu des bouleversements touchant le sous-sol du monument.

Les nombreuses interventions archéologiques menées dans le cloître ont souvent apporté autant d'éléments nouveaux qu'elles ont soulevé de nouvelles questions ${ }^{1}$. Suite à la synthèse réalisée par $\mathrm{O}$. Ginouvez (Ginouvez 2000), il est aujourd'hui possible de mieux appréhender le contexte et les modalités de la superposition relative des cloîtres évoquée plus haut ${ }^{2}$. Toutefois, il restait plusieurs points d'incertitude et parmi ceux-ci deux sont directement concernés par l'opération que nous avons menée en décembre $2004^{3}$ :

* Contractuel, Ville de Caunes-Minervois. 54 rue de La Clamoux 11000 Carcassonne. arnaud.gaillard@free.fr

${ }^{1}$ Nous n'exposerons pas ici l'historique des recherches archéologiques dans l'abbaye ; cet aspect étant largement développé par O. Ginouvez (Ginouvez et alii 2010).

${ }^{2}$ La plupart des faits et interprétations de ce présent article font référence au travail de synthèse effectué par O. Ginouvez (Ginouvez 2000), (Ginouvez et alii 2010). Nous avons également exploité l'étude documentaire réalisée en 2003 par A. Béa (Béa 2003).

${ }^{3}$ Opération INRAP (Gaillard 2005) pour la quelle nous avons bénéficié de l'aide de Maxime Guillaume et Olivier Ginouvez que nous remercions vivement. 


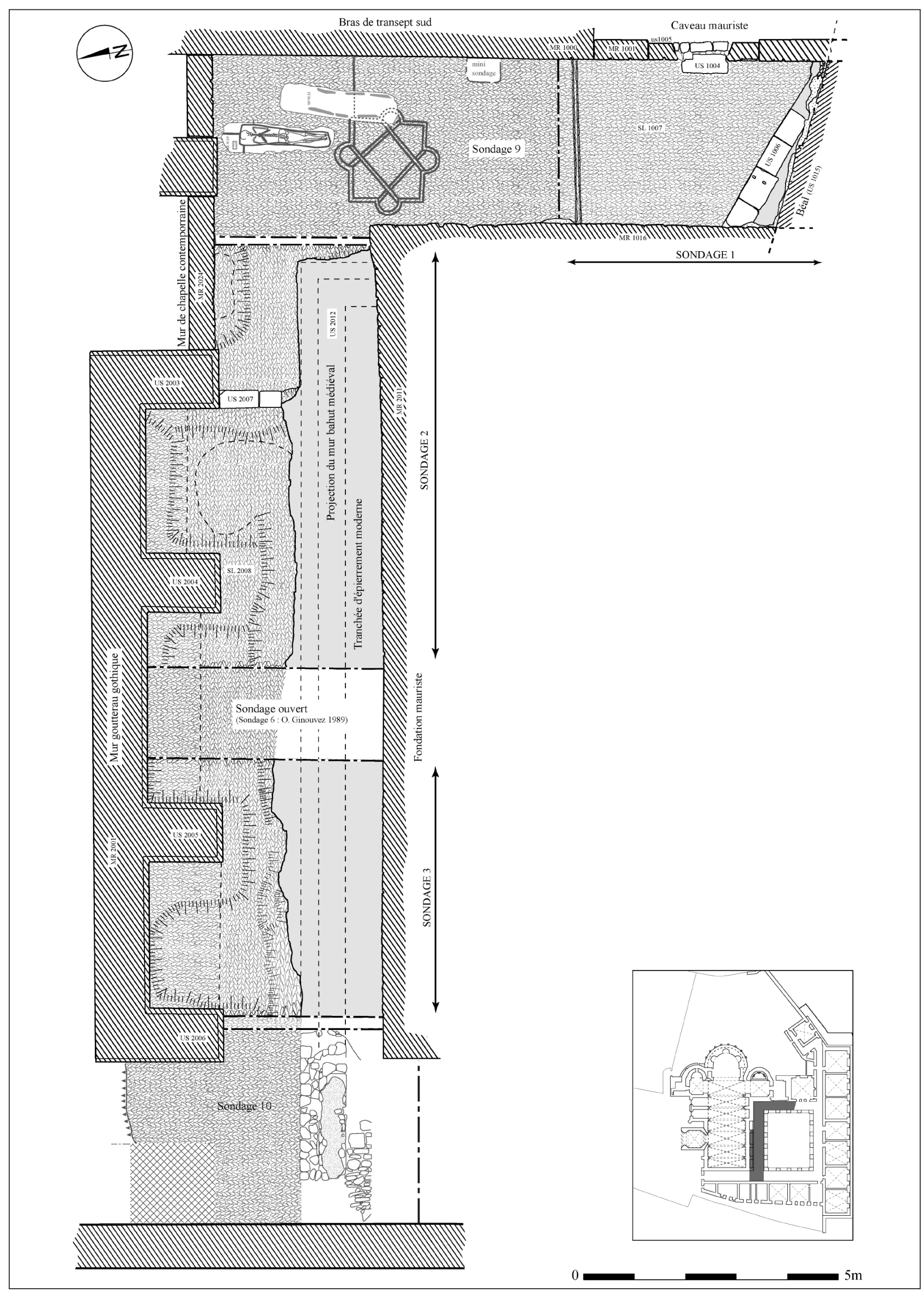

Fig.1 : Abbaye de Caunes-Minervois (Aude) : Cloître. Plan synthétique des galeries septentrionale et orientale (éch. 1 : 100) (D.A.O. : A. Gaillard). 


\section{- Le cloître médiéval : roman ou gothique?}

La découverte du sol en épis situé à près de 2,40 m au-dessous du cloître actuel a permis de confirmer l'existence d'un cloître plus ancien. Sa datation fine en l'état des interventions était peu aisée. La facture médiévale du sol en épis pouvait toutefois être mise en avant. La seule documentation dont nous disposions sur ce cloître était la gravure du Monasticon Gallicanum (1687) et une description de son état au XVII ${ }^{\mathrm{e}}$ siècle ${ }^{4}$.

\section{- Le cloître médiéval : quel plan et quelles dimensions?}

Les galeries nord et est du cloître médiéval ayant été mises au jour (sondages 5, 6, 9 et 10), deux sondages ont été réalisés afin d'appréhender la galerie sud (7 et 8). En l'état des fouilles, l'emplacement de cette galerie n'a toujours pas été découvert. La galerie ouest, quant à elle semble se situer au-delà du parement est de l'aile ouest des bâtiments conventuels du XVII ${ }^{\mathrm{e}}$ siècle. Un plan d'ensemble du cloître médiéval n'était donc pas réalisable en l'état.

\section{UN CLOITRE ROMAN ?}

A ce jour, nous ne disposons que de peu d'éléments permettant d'envisager l'existence d'un cloître antérieur au cloître que nous nommerons plus bas, le cloître gothique. Toutefois, certains indices nous laissent penser qu'un tel cloître a pu exister.

\section{Le mur gouttereau sud}

L'hypothèse de l'existence d'un mur gouttereau sud roman et donc d'une nef romane antérieure à l'actuelle, s'appuie essentiellement sur deux arguments et un indice plus ténu.

Le premier argument est relatif aux observations architecturales effectuées par M. Durliat : « La nef, refaite au $\mathrm{XIV}^{\mathrm{e}}$ siècle, mais en conservant le plan de la construction romane et une partie de son mur septentrional, comprend six travées extrêmement étroites et une septième à l'est un peu plus large, alignée sur les croisillons du transept bas » (Durliat 1987, 20). Le deuxième élément correspond aux observations effectuées dans le sondage 3 par O. Ginouvez en 1989 (Ginouvez et al. 1989, 124-128 et Ginouvez 2000, 24). Ce sondage a révélé la présence d'un mur massif dérasé (MR3107) interprété comme étant probablement le vestige d'un mur gouttereau roman servant de fondation à l'actuel mur gouttereau. Enfin, ce qui apparaît comme étant une série d'assises débordantes des contreforts de la galerie nord (cf : $I I-1$ ) pourrait être interprété comme étant des contreforts plus anciens et plus larges.

L'ensemble de ces éléments viendrait globalement corroborer l'hypothèse émise par M. Durliat. Ainsi, l'angle formé par ce mur gouttereau et par le bras de transept sud a pu en toute logique accueillir l'angle nordest d'un premier cloître.

\section{L'aile est de la galerie est}

Le seul élément architectural indubitablement roman ${ }^{5}$ que nous ayons pu observer est le bras de transept sud. Lors du terrassement mécanique de la galerie est, le mur ouest de ce bras de transept sud a été dégagé. Il s'agit d'un mur en grand appareil calcaire allogène de bonne facture $^{6}$. Des dalles d'ardoises sont employées pour ajuster les niveaux d'assises.

Dans l'alignement de ce mur, un pan de mur non harpé au précédent ( « coup de sabre ») est constitué d'un appareil similaire (MR1001). Les assises ne sont pas au même niveau, toutefois il semble que ces deux murs appartiennent à la même entité architecturale ; Ce mur présente également des dalles d'ardoise aux interstices. A environ 1,20 m. du « coup de sabre » se trouve une ouverture en plein cintre aménagée dans le mur MR1001.

L'arc en plein cintre est constitué de 10 claveaux. La largeur au sol de cette ouverture est de $208 \mathrm{~cm}$. Sa hauteur par rapport au sol en épis est de $184 \mathrm{~cm}$ (fig. 2).

Un seuil fonctionnant avec cette ouverture est constitué de deux larges blocs calcaires taillés et jointifs. Un surcreusement au centre du seuil matérialise un emmarchement. Ce seuil (us 1003), probablement roman, se situe à près de $20 \mathrm{~cm}$ au-dessous du niveau de la calade et devait donc fonctionner avec un niveau de sol plus profond. Toutefois, le sondage perforant la calade effectué par J. P. Caser (Caser 1988) au droit du bras de transept, n'a pas permis la reconnaissance de ce niveau.

\section{Un mur bahut roman?}

Nous sommes tentés, toujours à titre d'hypothèse, d'évoquer dans ce chapitre le mur bahut médiéval en partie démantelé et observé dans le sondage 10 en 1989 (Ginouvez et al. 1989). Ce mur (MR10112 ; Ginouvez 2000,31 ) a été mis au jour sur une longueur de 2,45 m. Sa largeur est de 0,54/0,55 m et son élévation est conservée sur une hauteur de $0,36 \mathrm{~m}$. Le flottement chronologique actuel le concernant pourrait permettre de le rattacher à une phase ancienne de construction (antérieure aux aménagements gothiques). Si l'on reprend

\footnotetext{
${ }^{4}$ A.D. 11 : H 1 - Abbaye de Caunes (bénédictins), registre des arrêts, contrats et autres actes du monastère, depuis le 3 juin 1659 jusqu'au 24 mai 1747. Bâtiments conventuels. 1664, 13 janvier. Etat des lieux.

"Il n'a aucun lieu régulier en estat que le Chapitre, qui est tout au fonds de l'Esglise, nouvellemant recouvert. Tout le cloître est descouvert ; mesme tous les pilliers en pierre des arceaux manquent du costé du couchant, et plus de la moitié du costé de l'église. Ce qui estoit probablemant anciennemant le réfectoire n'a que les quatre murailles, sans couvert, et la muraille du costé du levant manque au bastimant qui est ensuitte, et les pierres aussi de la muraille. Les autres murailles sont fort corrompues."

${ }^{5}$ DURLIAT 1988 : «Au cours du XII ${ }^{\mathrm{e}}$ siècle, furent construits les deux bras d'un transept bas greffés d'une absidiole semi-circulaire. Les travaux commencèrent par le nord et s'achevèrent au sud, avec un bras et une absidiole plus conséquents. La voûte suit une forme rare combinant voûte d'arêtes et coupole ».

${ }^{6}$ cf. : mr 9101, Ginouvez 2000.
} 


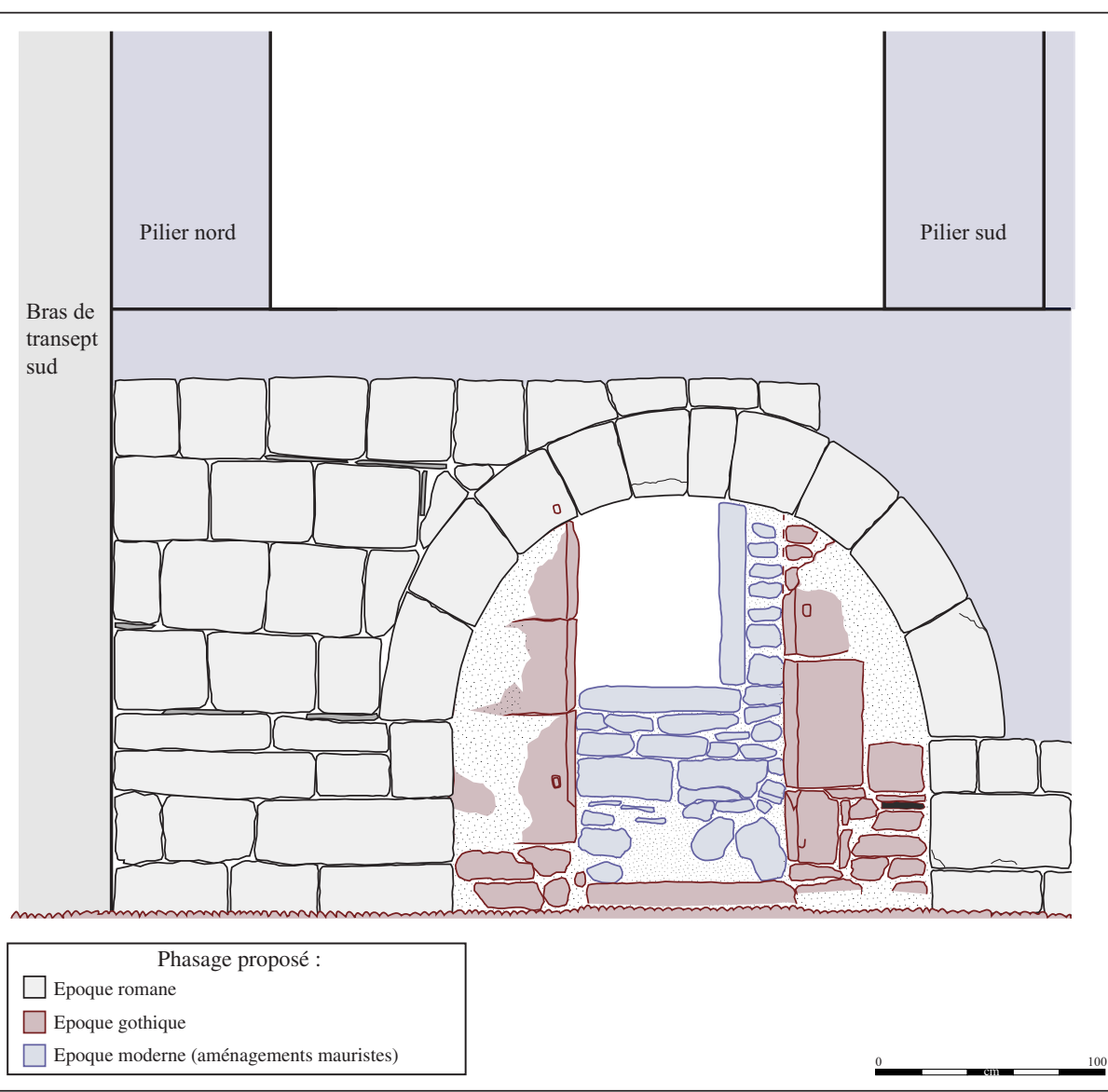

Fig. 2 : Abbaye de Caunes-Minervois (Aude) : Cloître. Elévation de la galerie orientale (D.A.O. : A. Gaillard).

l'hypothèse d'une nef romane légèrement plus étroite (cf. : sondage 3, Ginouvez 1989 et 2000), nous pouvons supposer qu'un cloître y ait été accolé (cf. I - 1). Ainsi, l'idée de la conservation d'un mur bahut roman existant (et des arcatures qui s'y appuient) lors des aménagements gothiques nous paraît envisageable.

\section{LE CLOITRE GOTHIQUE}

\section{Le sol en épis}

Le sol en épis déjà observé à maintes reprises (Caser 1988, Ginouvez 1989 et Gaillard 2001) est conservé sur pratiquement toute la surface fouillée. Ce sol est composé de galets calibrés « posés sur la tranche suivant un motif en épis » (Ginouvez 2000, 35).

Dans la galerie est, seule la bande de contact entre l'alignement de dalles et le béal est exempte de galets. Cette zone (us 1017) correspond vraisemblablement à la tranchée de fondation du béal et ne nous permet pas par conséquent, de savoir si le sol en épis continuait au-delà des dalles, vers le sud.

Le sens des épis suit la galerie (axe N / S) sur toute la longueur fouillée. Les galets s'appuient sur le bras de transept sud et le mur qui est dans sa continuité (MR 1001). Les assises du mur bahut moderne recouvrent le sol en épis et il est probable que la tranchée de fondation de ce mur l'ait perforé.
Dans la galerie nord, seule une bande très étroite (20 cm environ) couvre toute la largeur de la galerie actuelle à l'extrémité orientale du secteur 2. La calade a été mise au jour entre les contreforts et couvre prés de la moitié de la surface comprise entre les contreforts et le mur bahut moderne. L'orientation des épis est N / S pour les parties comprises entre les contreforts et $\mathrm{E} / \mathrm{O}$ pour la calade située dans la galerie. Le changement de sens des épis s'effectue exactement au droit du quatrième et du cinquième contrefort. Cette limite est en léger retrait entre le troisième et le quatrième contrefort, tandis que le retrait est plus prononcé entre le second et le troisième contrefort. Les galets s'appuient directement contre le mur gouttereau sud (mr 2001) et les contreforts. Aucun joint au mortier ou reprise de la calade n'a été observé. Nous en déduisons donc que ce niveau de sol est postérieur à la reconstruction gothique de la nef et des contreforts.

Le creusement de la tranchée d'épierrement du mur bahut médiéval MR 2010 (cf : III - 2) a eu des conséquences de deux ordres sur ce sol en épis :

- Immédiates et à court terme : La plupart des galets en bord de calade sont soit déstabilisés, soit déchaussés : Ce sont seulement les trois mètres les plus à l'est qui permettent d'observer précisément ce qui fut la limite entre la calade et le mur bahut médiéval. A l'extrémité occidentale de la portion fouillée, nous pouvons constater un phénomène d'effondrement de la bordure de 
calade. Sur cette partie, les galets ne sont plus de champ mais couchés sur leur face latérale sud.

- A plus long terme : La décompression des épis et des niveaux sous-jacents à entraîné d'importants affaissements de la calade. Ainsi, nous pouvons observer un phénomène de soutirage des niveaux d'assise de la calade entre chaque contrefort. Le sol en épis ne semble avoir conservé son altitude initiale que sur les parties étroites jouxtant le mur gouttereau et les contreforts. Autour de ceux-ci, la calade dessine à chaque fois une forme quadrangulaire qui pourrait correspondre à un large degré de fondation des contreforts ou les fondations de contreforts plus anciens sur lesquels la calade aurait été bâtie. Cette question a déjà été soulevée au vu d'un élément bâti analogue observé par $\mathrm{O}$. Ginouvez dans le sondage 9, sous la sépulture SP 9118 ayant crevé la calade (cf. : fig. 13 et p. 30, Ginouvez 2000 : MR 9119).

Il semble malgré tout que le démantèlement du mur bahut médiéval n'ait pas affecté l'entendue initiale du sol en épis. De ce fait les limites de la tranchée paraissent nous renseigner assez précisément sur le retour du mur bahut médiéval vers la galerie est. Ce retour situerait donc l'emplacement du mur bahut médiéval de la galerie est, sensiblement au même endroit que le mur bahut actuel. La galerie médiévale est aurait alors autour de quatre mètres de large (entre le bras de transept et ce retour) contre $1,60 \mathrm{~m}$ pour la galerie nord (espace compris entre les contreforts et la projection du mur bahut observé dans le sondage 10). Cette disproportion déjà observée (Ginouvez 2000, 31) est particulièrement délicate à interpréter tant les inconnues sont nombreuses (emplacement précis du gouttereau sud roman, existence et taille des contreforts associés...). Nous pouvons malgré tout supposer que les choix architecturaux faits à l'époque gothique aient été fondés sur un consensus entre le bâti existant et les aménagements à faire, ceci au dépit d'une unité architecturale.

\section{Aménagements de la calade et éléments décoratifs}

Dans la galerie est, un alignement de quatre dalles (us 1006) contient directement la pression du sol en épis (sl 1007) au sud de l'ouverture du caveau. Le sol s'interrompt brutalement dans l'alignement des dalles et suggère l'existence de dalles analogues aujourd'hui disparues (démontage probable lors de l'aménagement du béal). Les éléments encore en place sont soigneusement ajustés et dessinent un axe oblique par rapport à l'ensemble des éléments architecturaux visibles. Les deux dalles les plus à l'est sont des marches réemployées (présence d'une zone polie et une zone piquetée), la troisième présente deux creusements quadrangulaires (ancrages de gonds ?) et semble donc être aussi un élément réemployé. La dernière dalle n'est visible que très partiellement ; les assises du mur bahut moderne mr 1016 la recouvrent presque entièrement.

La fonction de cet aménagement nous a semblé être initialement celle d'un drain. Toutefois, un mini sondage de $40 \mathrm{~cm}$ de profondeur pratiqué au droit de la dernière dalle et dans la tranchée d'épierrement des dalles est, nous permet de remettre largement en question cette première interprétation.

La dalle observée en coupe a $42,5 \mathrm{~cm}$ de largeur et est épaisse de $21,5 \mathrm{~cm}$, elle repose sur :

- Un premier lit (4 cm d'épaisseur) de sédiment hétérogène tassé (déchets calcaires, gravillons, fragments de tuiles, nodules de mortier). Quelques tessons de céramique monochrome grise ont pu être observés.

- Un lit d'argile rouge compacte avec des ardoises (5 cm d'épaisseur). Stérile.

- Un niveau de sédiment (10 cm d'épaisseur) brun argileux et hétérogène (déchets de construction).

- Un niveau compacté hétérogène (nombreux galets et gravillons, quelques nodules de mortier).

L'absence de dalles de champ, supportant les dalles de couverture, l'absence de fil d'eau aménagé à près de $40 \mathrm{~cm}$ de profondeur, l'épaisseur des dalles et la nature des sédiments recouverts par celles-ci plaide plutôt pour un aménagement décoratif ou du moins, non hydraulique, reposant sur des niveaux de sol de chantier et/ou de circulation antérieurs (sols romans ?). L'obliquité de l'axe des dalles reste difficile à interpréter. Contrairement à l'orientation de la portion étudiée du béal (cf. : III - 5), aucune contrainte ne semble pouvoir être évoquée. S'agit-il d'un indice sur l'orientation de la galerie médiévale sud ou cette obliquité a une simple valeur décorative ? Nous pouvons également nous interroger sur l'éventuelle matérialisation d'un passage entre la galerie et une possible ouverture, sous l'actuelle salle capitulaire (cette ouverture aurait été reprise et agrandie par le béal). En l'état, nous ne sommes pas en mesure de répondre à ces questions.

Un bandeau composé de deux lignes de briquettes à cuisson oxydante posées de champ a été observé à $20 \mathrm{~cm}$ au nord du « coup de sabre ». Ce décor n'est pas parfaitement perpendiculaire au bras de transept (l'angle est plus fermé au sud). Les deux lignes de briquettes enserrent deux alignements de galets composant un épi. Cet épi est perpendiculaire au sens des épis environnants (orientés nord / sud). La facture de ce décor et les matériaux employés sont analogues à ceux observés à la jonction des galeries nord et est (Gaillard 2001 et paragraphe suivant). Il semble que ce bandeau matérialise la transition entre la galerie est et la galerie nord. Un tel aménagement n'a pourtant pas pu être observé entre la galerie nord et l'angle de celle-ci avec la galerie est. Toutefois, le décapage de la portion non fouillée comprise entre les limites de ce présent sondage et le sondage couvert (Gaillard 2001), pourra peut-être nous éclairer sur ce point.

L'opération de 2001 a permis le dégagement d'un motif aménagé dans la calade à l'extrémité nord de la galerie est (fig. 4). Un lobe de ce motif avait déjà été observé (Ginouvez 1989) : « une forme d'emblema constitué de deux demi-cercles opposés à deux triangles dépourvus de bases » (Ginouvez 2000, 38), sans que la forme complète soit déduite. Cette décoration se 
compose d'un carré dans lequel s'inscrit un autre carré. Les angles de ce carré sont encadrés de lobes débordants le premier quadrilatère au centre de chacun de ses segments. Ces figures sont élaborées à partir de briquettes jointives, posées de chant, dessinant des lignes parallèles. L'espace compris entre ces lignes est décoré de galets en épis dont l'orientation est contingentée par le dessin des briquettes, indépendamment du sens général des épis du sol environnant. Une ligne simple de briquettes, fait la jonction entre la décoration et le mur du croisillon nord. Les équerres de blindage de coupe ne permettent pas d'observer si une ligne similaire existe à l'ouest du motif. L'hypothèse d'une ligne continue entre le mur du transept et le mur bahut du cloître primitif est envisageable ; cette ligne matérialiserait le début de la galerie est du cloître roman. D'après la position hypothétique de l'angle nord-est du mur bahut médiéval, proposée par O. Ginouvez (Ginouvez 1989 et 2000), il paraît vraisemblable que ce motif ait une position centrale dans la largeur de la galerie médiévale. De même, nous pouvons penser que chacune des galeries médiévales présente à chaque angle ce type de décoration.

Dans la galerie nord, un alignement de deux dalles calcaires (us 2007) a également été mis au jour dans l'alignement précis du parement occidental du deuxième contrefort. Ces dalles de même largeur, sont ajustées avec soin et contiennent directement les galets du sol en épis. Une troisième dalle devait probablement compléter cet alignement au sud, jusqu'au mur bahut médiéval. La vocation de ces dalles est probablement décorative et nous sommes tenté d'effectuer un parallèle avec les dalles observées dans la galerie est (us 1006).

\section{Eléments de datation du sol en épis}

Les éléments permettant de caler chronologiquement le sol en épis sont ténus puisque aucune mention écrite ne vient nous éclairer sur ce point. Cependant, plusieurs hypothèses ont été émises depuis sa mise au jour. Ainsi, O. Ginouvez évoquait la possible antériorité de la calade par rapport à la reconstruction gothique, mais n'excluait pas l'inverse (Ginouvez 2000, 35).

A ce jour, les indices basés sur la chronologie relative dont nous disposons sont plus nombreux :

Dans la galerie nord, la calade s'appuie sur le mur gouttereau sud et ses contreforts.

Les changements de direction des épis semblent être en partie conditionnés par la présence des contreforts (cf. entre le $4^{\mathrm{e}}$ et le $5^{\mathrm{e}}$ contrefort).

La pose des dalles est très certainement faite en fonction du parement du deuxième contrefort.
Le démantèlement du mur bahut médiéval a entraîné un phénomène de soutirage permettant d'observer que la pose de la calade s'effectue au-dessus des fondations des éléments d'architecture gothique.

La calade semble donc postérieure à cette élévation gothique qui est datée par M. Durliat du XIV ${ }^{\mathrm{e}}$ siècle sans plus de précisions 7 . Toutefois, une mention récemment exhumée des Archives Nationales (Béa 2003), donne un jalon chronologique supplémentaire ${ }^{8}$ et permettrait de situer la construction de la calade dans la première décennie du XIV ${ }^{\mathrm{e}}$ siècle. Ainsi, la proposition d'une calade romane nous semble donc peu viable.

En ce qui concerne la chronologie relative, la pose de la calade dans les deux galeries semble relever de la même campagne de travaux.

\section{Réduction de l'ouverture de la façade est, galerie est}

Deux piédroits accolés (blocs en réemplois) réduisent l'ouverture initialement en plein cintre (us 1005). Ces éléments reposent directement sur le seuil probablement roman précité (cf : $I-2$ ) et contiennent un emmarchement constitué de blocs équarris et d'une dalle liés au mortier blanc (fig.2 et 3). Ce mortier scelle également les galets du sol en épis qui présente un léger renflement au contact de cet emmarchement. La fonction de cette réduction paraît être liée à une volonté d'installer une porte à double battant de dimension plus modeste (présence d'ancrages de gonds dans les piédroits). La vocation de la salle ainsi close semble toujours correspondre à un espace transition et de circulation entre l'église et le cloître. La datation de cet aménagement est peu aisée; le seul élément de chronologie relative tangible dont nous disposons est le scellement au mortier de l'emmarchement et du sol en épis. Nous en déduisons une relative contemporanéité entre la pose de ce sol et la réduction de l'ouverture.

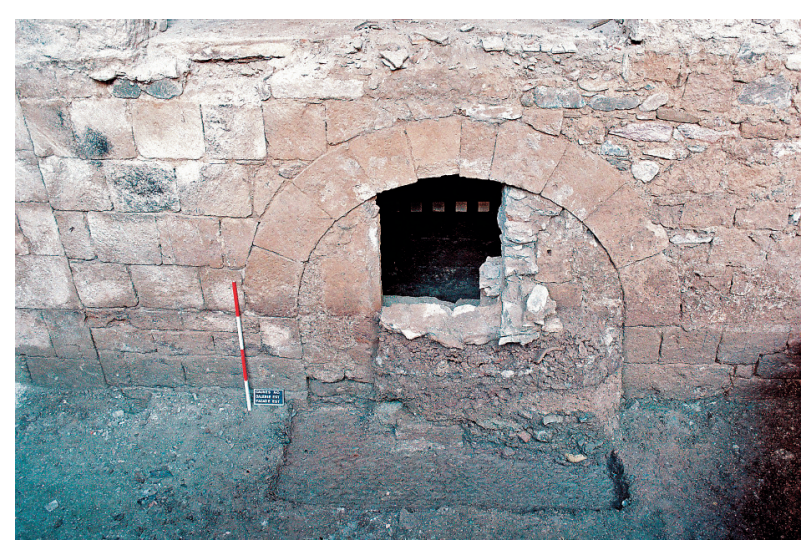

Fig. 3 : Abbaye de Caunes-Minervois (Aude) : Sondage 1, façade orientale en cours de dégagement (Photo : A. Gaillard).

\footnotetext{
${ }^{7}$ Durliat 1988, 20 : «La nef, refaite au XIV ${ }^{\mathrm{e}}$ siècle, mais en conservant le plan de la construction romane et une partie de son mur septentrional, comprend six travées extrêmement étroites et une septième à l'est un peu plus large, alignée sur les croisillons du transept bas ».

${ }^{8}$ B.N.F. : Fonds DOAT, Vol. 58 - Diocèse de Narbonne, Abbaye de Caunes. Ordre de saint Benoît. Depuis 787 jusqu'en 1468. (Cf Fonds Doat, Vol. 3 , $\left.\mathrm{f}^{\circ} 190 \mathrm{v}^{\circ}\right)$. " $\gg$ Bulle du pape Clément 5 e par laquelle il accorde un an et quarante jours d'Indulgence a ceux qui visiteroient chaque annee les jours de festes de S. Pierre et S. Paul l'Eglise Abbatialle de Caunes fondée a l'honneur desd. saincts 4e kalendas Martii Pontificatus anno 4e. [1308] ».

D'après A. Béa, cette dotation d'indulgences pourrait permettre de dater l'achèvement de la nef.
} 


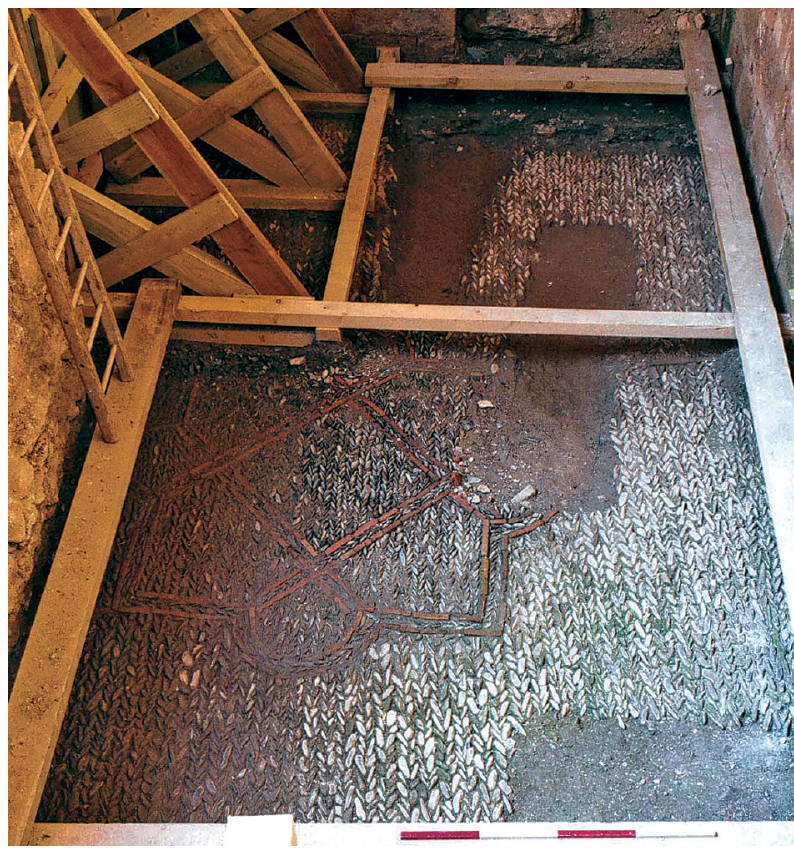

Fig. 4 : Abbaye de Caunes-Minervois (Aude) : Galerie orientale du cloître : décor de briquettes et calade (Photo : A. Gaillard)

\section{AMENAGEMENTS MODERNES}

\section{Le niveau argileux}

La phase de remblaiement moderne repose sur un niveau d'argile rouge et de plaques de lauzes posées à plat scellant directement le sol en épis. Cette couche a été observée sur l'ensemble des portions fouillées (galeries est et nord). Elle est dans l'ensemble d'épaisseur relativement régulière (20-30 cm environ). Le rare mobilier observé dans ce niveau se compose de tessons très fragmentés de céramique à pâte oxydante et glacis beige, vraisemblablement moderne, comme le propose O. Ginouvez : « ...peut être datée du courant des XVI ${ }^{\mathrm{e}}$ XVII ${ }^{e}$ siècles » (Ginouvez 2000, 41).

La fonction de ce niveau, reste peu claire, nous pouvons toutefois avancer deux hypothèses qui ne sont pas exclusives :

- L'hypothèse d'un nivellement préalable aux travaux de construction des murs bahuts. Dans ce cas, il s'agirait d'un sol de travail permettant le passage d'engins à roues (tombereaux...).

- L'hypothèse d'une tentative d'étanchification du sol par l'emploi d'argile et de lauzes afin d'éviter les remontées d'eau.

Notons enfin que la présence massive d'ardoises taillées, ayant parfois $45 \mathrm{~cm}$ de long, nous évoque un réemploi probable des éléments de couverture des galeries médiévales.

Dans la galerie nord, le niveau d'argile rouge plastique (us 2009) a été reconnue sur toute la longueur de la galerie. Cette bande, immédiatement sous-jacente aux premiers remblais, ne couvre que la zone en épis ; la portion correspondant à la tranchée d'épierrement du mur bahut médiéval est directement recouverte de remblais modernes (cf. : $I V-2$ ). Les caractéristiques de ce niveau sont analogues à la couche argileuse observée dans la galerie est (us 1008). Elles permettent d'envisager une relative contemporanéité des deux niveaux (même phase d'aménagement légèrement antérieure à la construction des murs bahuts modernes).

\section{La tranchée d'épierrement du mur bahut médiéval}

La tranchée d'épierrement du mur bahut médiéval (us 2010), dans la galerie nord, a déjà été observée lors de la fouille du sondage 6 (Ginouvez 1989). Faute de mur bahut, nous avons le témoignage de son existence par cette tranchée. Celle-ci a par endroit, déstabilisé ou entamé les derniers galets du sol en épis (cf : II - 1). Le comblement de cette tranchée (us 2012) est composé de sédiments hétérogènes chargés en débris de construction (nodules de mortier et fragments de blocs équarris) comparables aux niveaux supérieurs (us 2013). Le mobilier mis au jour est également moderne (tessons de céramiques vernissées...).

Il semble que l'épierrement du mur bahut médiéval ait été rendu nécessaire par la proximité des fondations du nouveau mur bahut. Enfin, le fait que le niveau argileux rouge vienne en limite de cette tranchée corrobore à nos yeux l'hypothèse d'un sol de travail évoqué plus haut (cf. : III -1 ) pour ce niveau argileux.

\section{Les clôtures des contreforts}

Deux dispositifs de clôture des niches situées entre les contreforts de la galerie nord ont pu être étudiés. Ces aménagements se situent entre le quatrième et le cinquième contrefort et entre le cinquième et sixième contrefort. Un même dispositif a été observé par O. Ginouvez en 1989 (sondage 6) entre le quatrième et troisième contrefort.

Dans tous les cas, un alignement de claveaux réemployés est aménagé au droit des contreforts et repose sur le niveau argileux décrit plus bas. Les claveaux sont en calcaire et semblent provenir d'un même édifice ; leur largeur est de $49 \mathrm{~cm}$ et la plupart présente sur l'intrados, une extrémité sculptée en boudin et l'autre en creux. Ces éléments sont posés de champ sans liant. La partie incurvée des claveaux (intrados) est face au mur bahut. Suite à la dépose des claveaux, il a été reconnu une continuité stratigraphique entre les remblais de la niche et les remblais de la galerie. Nous en concluons que cet aménagement intervient en cours de remblaiement, postérieurement aux premiers dépôts de sédiments.

Quant à la fonction de ce dispositif, l'hypothèse avancée par $\mathrm{O}$. Ginouvez : « ... un degré destiné à porter un autel pariétal ou une statue de culte » (Ginouvez 2000,41 ) nous semble envisageable, bien que son recouvrement paraît s'effectuer rapidement.

\section{Le caveau mauriste}

Cette structure a été fouillée par Jean-Pierre Caser entre 1990 et 1991. Il s'agit actuellement d'une pièce 
souterraine occupant l'espace compris entre le bras de transept sud et le mur nord de l'actuelle salle capitulaire qui fait office de «musée lapidaire ». Cette pièce (cf : II - 4) a été couverte à l'époque moderne par une voûte appareillée de profil surbaissé qui fonctionne comme un pont $^{9}$ car non harpée aux murs latéraux (mur soutenant le palier de l'escalier à l'est et mur dans la continuité du bras de transept à l'ouest) et reposant sur deux piles adossées aux murs nord et sud. Les joints au mortier de cette voûte sont rehaussés d'un trait ocre.

En façade, une maçonnerie vient obturer l'espace compris entre les deux piédroits gothiques (cf : II -4) jusqu'à mi-hauteur, puis sur une bande verticale accolée au piédroit sud. Cet aménagement est composé de blocs peu ou non équarris agencés avec peu de soin et liés au mortier gris. Une dalle horizontale est posée sur la partie inférieure, elle soutient une dalle verticale scellée à l'aménagement vertical. Ces dalles semblent destinées à contenir l'escalier du caveau moderne.

En effet, l'accès à cette pièce était rendu possible après remblaiement par l'aménagement d'un escalier étroit dont l'ouverture était obturée par une dalle de marbre situé en surface de la galerie est. Lors du décapage mécanique de la galerie est, il a été décidé d'effectuer une coupe à l'aplomb du parement nord de cet aménagement afin d'en mieux comprendre la chronologie. Les premières assises de l'escalier reposent directement sur une couche de remblais dont l'inclinaison est non seulement conservée (absence d'aplanissement), mais utilisé pour asseoir le pendage des marches. Les parements de l'escalier sont peu soignés ; Ils sont composés de blocs de remploi et de galets liés par un mortier gris. Les parements et les dernières marches de l'escalier ne sont pas harpés à la façade est du caveau, ils pénètrent une ouverture aménagée et reposent sur les remblais. Il semble donc que cet aménagement intervienne en cours de comblement de la galerie.

Un aménagement moderne de la façade est également visible. En effet, la partie supérieure du mur roman (us 1010) situé dans la continuité du bras de transept est constituée de blocs de remploi et de galets, de facture plus sommaire, liés au mortier gris. Cette reprise est nettement postérieure à l'aménagement de l'arc en plein cintre et semble correspondre à la phase d'aménagement du palier actuel et de l'arche supportant l'escalier menant aux étages du clocher sud.

\section{Le beal}

Cette canalisation couverte (us 1015) a pour fonction de récolter et évacuer les eaux (pluviales et usées) en amont, elle appartient à un réseau complexe contournant le parvis de l'abbatiale par l'ouest, traversant le cloître, l'aile orientale des bâtiments conventuels et les jardins pour se connecter à un autre beal d'axe nord / sud, à l'extérieur de l'enclos abbatial.
Il semble que l'on doit cet aménagement à la Congrégation de Saint-Maur, qui à la fin du XVII ${ }^{e}$ siècle, entreprend la mise hors d'eau de l'abbaye par l'exhaussement des architectures ; la présence du beal complèterait ainsi ce dispositif en drainant les eaux souterraines.

Cette construction massive traverse le cloître moderne en fonction d'un axe globalement est / ouest (fig. 5). Deux regards sont visibles dans la galerie ouest et un troisième est mentionné au centre de la cour du cloître. Son tracé dans le cloître est rectiligne jusqu'à la jonction avec les fondations du cloître moderne de la galerie est ; un coude oriente à ce niveau le beal vers le sud.

La portion observée traverse la galerie est. En élévation, le mur du cloître s'appuie sur l'extrados de la voûte et un joint au mortier scelle ces deux structures. Le beal perfore le mur en appareil calcaire (MR 1001) sans qu'un joint n'ai été entreprit. Le niveau de sol en épis, interrompu par un alignement de dalles, est perforé par les fondations du beal. Ces dernières scellent en partie la dernière dalle en place (cf. : $I I-2$ ). Un regard aménagé postérieurement à la construction du béal est visible à $50 \mathrm{~cm}$ du mur est.

L'orientation du beal est difficile à interpréter : l'ensemble des éléments modernes observés en élévation semble orthonormé, hormis la terminaison de l'aile orientale des bâtiments conventuels. Le coude du beal semble donc être la réponse à une contrainte. Sa construction est très probablement antérieure à l'élévation du cloître actuel, il est donc probable que les bâtisseurs avaient une vision exhaustive des éléments architecturaux en place (retour de la galerie sud du cloître médiéval, bâtiments accolés...). Deux hypothèses peuvent alors être avancées parmi ces possibles contraintes ; la nature du terrain (socle rocheux...) et la topographie des lieux sur la partie sud (déclivité, fossé, enceinte...), liée à la proximité de l'Argent-Double comme le suggère $\mathrm{O}$. Ginouvez (Ginouvez 2009). Une dernière, plus pragmatique, considère que la déviation de l'axe du béal est réalisée afin d'éviter la perforation du

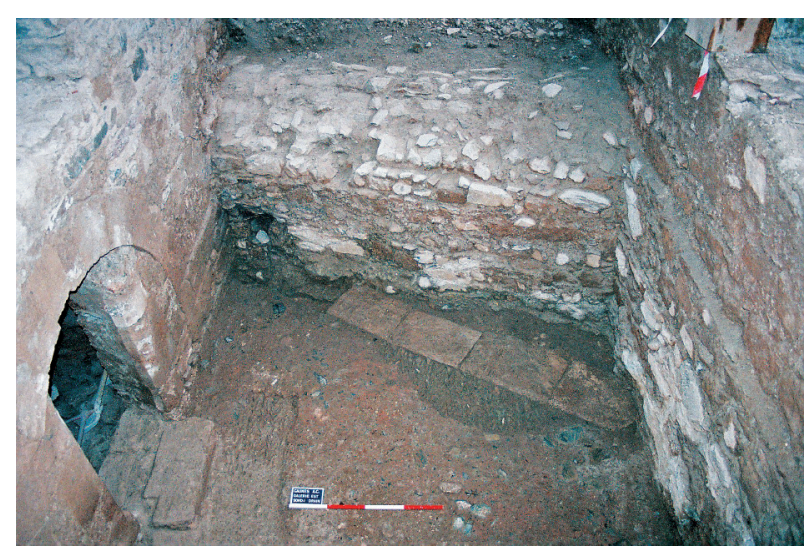

Fig. 5 : Abbaye de Caunes-Minervois (Aude) : Tronçon du beal visible dans la galerie orientale (Photo : A. Gaillard).

${ }^{9}$ Communication orale de Monsieur Régis Martin (ACMH) que nous remercions. 
mur sud de la tour clocher, lui-même orienté est / ouest et particulièrement massif. En ce qui concerne la date de construction du beal, il semble qu'elle soit antérieure à $1669^{10}$.

\section{LE CLOITRE MODERNE}

\section{Les fondations du cloître}

Les fondations modernes observées dans les galeries est et nord sont particulièrement massives. O. Ginouvez les qualifient de «puissantes longrines » ayant près de 3,30 $\mathrm{m}$ de large pour les parties basses étudiées et présentant deux ressauts sur les parements internes auxquels semblent répondre deux ressauts observés sur le parement externe (cour du cloître). Ces murs sont constitués d'un appareil irrégulier "mêlant moellons bruts, moellons dressés et, localement pierre de taille en moyen appareil. Les remplois sont nombreux. " (Ginouvez 2000, 43). La base de leur fondation n'a jamais pu être observée en raison des remontées d'eau. La profondeur maximale observée est 3,30 m (Ginouvez 2000, 42). Leur élévation a impliquée le creusement d'une large tranchée de fondation pour la galerie nord alors que dans la galerie orientale, les fondations du cloître moderne semblent perforer le sol en épis à son aplomb.

\section{La phase de remblaiement}

La dynamique de remblaiement des galeries du cloître a été observée à plusieurs reprises (Caser 1988 et 1990, Ginouvez 1989 et 2000, Gaillard 2001) dans les galeries Est et Nord. Malgré quelques variantes, ce remblaiement concerne l'ensemble du cloître et correspond à une phase importante de travaux dont l'objectif semble être la mise hors d'eau des bâtiments. Cette campagne de travaux correspond aux aménagements entrepris par la congrégation de Saint Maur à l'extrême fin du XVII ${ }^{e}$ siècle - début XVIII ${ }^{\mathrm{e}}$ siècle.

Dans la galerie est, ces remblais se composent de passes hétérogènes, majoritairement limoneuses. Trois phases principales ont été reconnues.

Une première série de passes forment un talus à fort pendage au droit des fondations du cloître.

Une seconde s'appuie sur le mur opposé afin d'inverser la pente.

Une dernière série de passes nivelle l'ensemble et supporte le dallage.

L'ensemble de ces passes semble déposé de l'intérieur du cloître vers l'extérieur. Un effet de « tombereaux versés » est d'ailleurs perceptible en coupe avec une multitude de variations de ces dépôts successifs.

Le mobilier observé dans ces remblais est constitué de matériel céramique de type médiéval lato sensu (céra- mique commune grise) mais également, et dans une plus grande proportion, de céramiques vernissées à cuisson oxydante (vernis jaunes, verts). Quelques éléments de faune ont été observés (fragments de diaphyses de petits et grands ruminants).

Toutefois, aucune concentration stratigraphique ou spatiale du mobilier n'a été remarquée. La présence concomitante d'éléments modernes et médiévaux dans l'ensemble des remblais n'a pas permis de distinguer des phases chronologiques distinctes de remblaiements et plaide plutôt pour un comblement rapide des galeries. La présence de mobilier, dont l'état de fragmentation est très variable, semble lié à des dépôts opportunistes de déchets culinaires. Quant à la présence d'éléments plus anciens, elle évoque plutôt le principe de dépôts en position secondaire.

Sont également présents dans ces remblais de nombreux déchets de constructions, blocs équarris, pierres de taille, nodules ou passes de mortier et fragments de tuiles. Dans la galerie nord, la dynamique de remblaiement observée entre le sol en carreaux de terre cuite et le niveau argileux coiffant le sol en épis est sensiblement identique à celle observée dans la galerie est. La seule variante réside dans la présence de passes obliques d'argile verdâtre, homogène et plastique, venant buter sur les contreforts. Le mobilier observé présente globalement la même proportion de tessons de céramiques grises médiévales mêlés à de plus nombreux tessons de céramiques vernissées jaunes et vertes. La présence de nodules de mortier et de déchets de construction est également à noter. L'absence de concentration du mobilier et la nature même des sédiments semblent indiquer une relative concomitance des remblaiements des galeries est et nord.

\section{AMENAGEMENTS POSTERIEURS A LA CONSTRUCTION DU CLOITRE MODERNE}

Des aménagements modernes et contemporains ont perturbé les niveaux superficiels du remblaiement ; Un dépôt d'ossements et deux sépultures en cercueil ont été observés à moins d'1,30 m de profondeur par rapport au sol de la galerie moderne. Un mur de chapelle contemporaine a également bouleversé ces niveaux.

\section{LA PISTE DE LA GALERIE MEDIEVALE SUD}

L'absence d'un éventuel retour de ce mur annonçant la galerie sud, est difficile à interpréter et laisse la porte ouverte à plusieurs hypothèses quant au plan du cloître médiéval :

Un mur bahut médiéval proche de l'actuel et épierré comme celui de la galerie nord.

Un mur bahut servant d'assise au béal et dessinant ainsi un cloître trapézoïdal très étroit.

\footnotetext{
${ }^{10}$ A.D. 11 : 4J 2/1 - Notes du chanoine Cals sur la commune de Caunes ( $2^{\mathrm{e}}$ cahier de $1^{\prime}$ abbaye, $\left.\mathrm{f}^{\circ} 8 \mathrm{r}^{\circ}\right)$. Monastère de Caunes. 24 avril 1772 . Transaction à propos de la prise d'eau : «L'établissement d'une digue ou d'un béal pour le côté opposé de la rivière de l'Argent Double au monastère ne doit être établi qu'à partir de celui du monastère et suivant et conformément à l'ordonnance de $1669 . .$. ».
} 
Un mur bahut plus distant, situé au-delà du mur actuel, dans la galerie sud du cloître moderne.

Cette dernière hypothèse nous paraît peu probable dans la mesure où les travaux de mise en place du drain ont également affecté l'intérieur de la galerie sud à une profondeur avoisinant les 4,20 mètres. Jusqu'à cette profondeur, aucune architecture n'a été observée lors de ces travaux effectués sans surveillance archéologique. De plus, un sondage réalisé dans la galerie sud s'est révélé négatif (cf. sondage 8, Ginouvez 1989). En l'état des connaissances nous ne pouvons que constater l'absence de retour et considérer l'hypothèse déjà émise par O. Ginouvez (Ginouvez 2010), d'un remploi de cette galerie pour réaliser le béal comme étant la plus plausible.

\section{PROPOSITION DE PHASAGE GENERAL (fig. 6)}

\section{PERIODE ROMANE :}

- Construction d'une nef légèrement plus étroite que l'actuelle (?) s'appuyant sur le bras de transept sud.

- Dans l'alignement de celui-ci : création d'une ouverture en plein cintre, avec un seuil soigné, permettant le passage entre le cloître, le chœur de l'abbatiale et probablement, la tour du Trésor.

- Création d'un cloître (?) adossé à ces éléments et dont le mur bahut médiéval épierré serait le témoin ?

\section{PERIODE GOTHIQUE :}

- Reconstruction d'un mur gouttereau sud avec contreforts, supportant une nef voûtée.

- Aménagement d'un sol en épis butant contre le mur bahut précité.

- Dans l'ouverture de la galerie est, mise en place d'un emmarchement. Réduction du passage par l'installation de piédroits permettant la pose d'une porte à double battant.

\section{PERIODE MODERNE :}

- Mise en place d'un sol de travail (niveau argileux) sur le sol en épis : Fin de fonctionnement du cloître gothique.

- Epierrement du mur bahut médiéval.

- Clôture des niches des contreforts dans la galerie nord (réemploi de claveaux).

- Dans la galerie est, transformation en caveau de l'espace accolé au bras de transept.

- Construction du béal.

- Construction des galeries du cloître moderne.

- Remblaiements et dallage : Cloître moderne fonctionnel.

- Inhumations superficielles dans les galeries (sépultures 1 et 2).

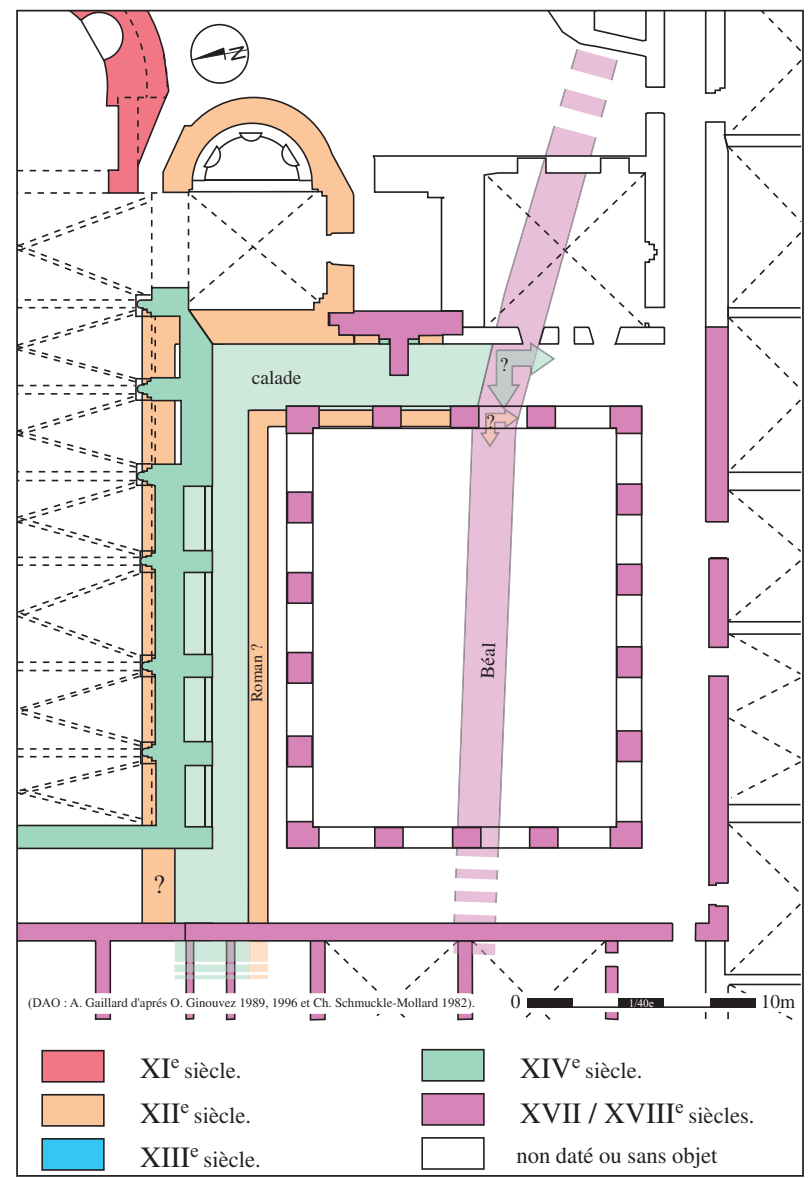

Fig. 6 : Abbaye de Caunes-Minervois (Aude) : Proposition de phasage chronologique des ensembles étudiés et hypothèses de développement du cloître médiéval (D.A.O. : A. Gaillard, d'après Ginouvez 1989, 1996 et Schmuckle-Mollard 1982).

\section{PERIODE CONTEMPORAINE :}

- Dépôt d'ossements dans la galerie nord.

- Aménagement d'un mur de chapelle entre deux contreforts.

\section{CONCLUSION}

Cette opération de fouille a permis de mettre au jour une grande partie de la galerie nord médiévale et une portion conséquente de la galerie est. Ces sondages associés aux précédents comprennent la quasi-totalité des parties du cloître médiéval comprises dans l'aire du cloître actuel. Pour autant, certaines questions restent toujours sans réponses.

Ainsi, nous ne sommes pas en mesure de localiser la galerie sud médiévale ; tout au plus, nous pouvons envisager de la situer globalement sous le béal.

La découverte d'un arc en plein cintre dans l'alignement du bras de transept sud nous paraît être un élément important. En effet, nous pourrons mener une réflexion sur les modalités de circulation entre le cloître, la tour sud et l'église à l'époque romane et peut-être définir la vocation initiale de l'espace réemployé en caveau à l'époque moderne. 
Cette ouverture associée à un premier seuil semble indiquer qu'un sol plus ancien (roman) a pu fonctionner avant la mise en place du sol en épis. Par conséquent, l'ancienneté du mur bahut médiéval en partie épierré, serait avérée.

En ce qui concerne la chronologie relative du site, les indices mis au jour nous permettent d'avancer que le sol en épis appartient à la phase gothique de reconstruction de la nef.

Gageons que les travaux de mise en valeur à venir permettront de nouvelles observations et pourquoi pas, d'apporter des éléments de réponse concernant le plan du cloître médiéval.

\section{BIBLIOGRAPHIE}

\section{- Etudes :}

Béziat 1880 : BEZIAT (L.), Histoire de l'Abbaye et de la ville de Caunes en Minervois. Paris, A. Claudin, 1880. 244 p.

Durliat 1970 : DURLIAT, (M.), L'église de Caunes-Minervois, in : Carcassonne et sa région. Actes des $\mathrm{XLI}^{e}$ et $X_{X I V}{ }^{e}$ Congrès d'Etudes Régionales. 1970, p. 59-63.

Durliat 1973 : DURLIAT (M.), L'église de Caunes-Minervois, Congrès Archéologique de France, 131 ${ }^{\mathrm{èm}}$ session, 1973, Pays d'Aude, Paris 1973, pp. 44-52.

Durliat 1986 : DURLIAT (M.), L'abbaye de Caunes. Monographie du CAML. Archéologie du Midi Médiéval, suppl. au t. $5,1986$.

Ginouvez et alii : GINOUVEZ (O.), CASER (J.-P.), SARRET (J.-P.), Interventions archéologiques dans les murs de l'abbaye bénédictine de Caunes-Minervois. Eglise et cloître méridional (1984-1991) : premier bilan, in : POUSTHOMIS-DALLE (N.), éd., L'abbaye et le village de Caunes-Minervois. Archéologie et histoire. Actes du colloque des 22 et 23 nov. 2003, Supplément nº 6 à A.M.M. Carcassonne, C.A.M.L., 2010, p. 37-55.

Griffe 1976 : GRIFFE (E.), Etudes d'Histoire Audoise (IX ${ }^{e}$ $X I V^{e}$ s. ). Carcassonne, 1976.

Mahul 1857-1871 : MAHUL (A.), Cartulaire et archives de l'ancien diocèse et de l'arrondissement administratif de
Carcassonne. Paris, 1857, I, p. 267 ; 1863, IV, pp. 67-135 et $166-187 ; 1867, \mathrm{~V}$, p. $163 ; 1871$, VI-1, pp. $115-116$, p. 179 , p. 204 , pp. $241-242$.

\section{- Rapport d'étude :}

Béa 2003 : BÉA (A.), Etude documentaire sur l'ancienne abbaye de Caunes-Minervois (Aude). Base de données informatique (FileMakerPro). 2003, 238 fiches.

\section{- Rapports de fouilles et synthèse :}

Caser 1990 : CASER (J.-P.), Sondage dans l'abbaye de Caunes-Minervois. 1990. Service Régional de l'Archéologie de Languedoc-Roussillon, 7 p., 19 pl.

Caser 1991 : CASER (J.-P.), Abbaye de Caunes en Minervois. Sondages 1991. Service Régional de l'Archéologie de Languedoc-Roussillon, 8 p., 16 pl.

Gaillard 2001: GAILLARD (A.), Abbaye de Caunes-Minervois (Aude). Réhabilitation des sondages ouverts du cloître. 2001. Service Régional de l'Archéologie de LanguedocRoussillon, 6 p., 4 fig.

Gaillard et coll. 2005 : GAILLARD (A.), PUCHEU LASHORES (C.), L'abbaye de Caunes-Minervois (Aude), Galeries nord et est du cloître. Rapport Final d'Opération - Fouille préventive. Service Régional de l'Archéologie de Languedoc-Roussillon, INRAP Méditerranée, Ville de Caunes-Minervois, 2005, $57 \mathrm{p}$.

Ginouvez et al. 1989 : GINOUVEZ (O.), LECUYER (N.), RINALDI (R.), Rapport d'étude, première partie : le cloître, 1989. Service Régional de l'Archéologie de Languedoc-Roussillon, 31 p., 20 fig.

Ginouvez et al. 1989 : GINOUVEZ (O.) et al., Caunes-Minervois, l'ancienne abbaye. Sauvetage, 1989. Service Régional de l'Archéologie de Languedoc-Roussillon, 65 p., 34 fig.

Ginouvez et coll. 2000 : GINOUVEZ (O.), GAILLARD (A.), Abbaye de Caunes-Minervois (Aude). Synthèse et observations critiques concernant les découvertes archéologiques réalisées entre 1984 et 1991 dans l'église abbatiale et le cloître. 2000, Service Régional de l'Archéologie de Languedoc-Roussillon, 65 p., 25 fig. 